\title{
Differences and Similarities in HIV Testing Among Men Who have Sex with Men and Women (MSMW) and Men Who Have Sex with Men Only (MSMO)
}

\author{
Cathy Maulsby ${ }^{*}, 1$, Frangiscos Sifakis ${ }^{2}$, Danielle German ${ }^{1}$, Colin P. Flynn ${ }^{3}$ and David Holtgrave ${ }^{1}$ \\ ${ }^{I}$ Department of Health, Behavior and Society and ${ }^{2}$ Department of Epidemiology, Johns Hopkins Bloomberg School of \\ Public Health, Baltimore, Maryland, USA \\ ${ }^{3}$ Infectious Disease and Environmental Health Administration, Maryland Department of Health and Mental Hygiene, \\ Baltimore, Maryland, USA
}

\begin{abstract}
The study examined differences in HIV testing between men who have sex with men only (MSMO) ( $n=300)$ and men who have sex with men and women (MSMW) $(n=105)$ and assessed associations with HIV testing among MSMW. A venue-based cross-sectional HIV surveillance study in 2008 (BESURE-MSM2) was examined. Prevalence of HIV testing was similar for MSMO and MSMW. One-on-one counseling (excluding counseling that is part of HIV testing) and having seen a health care provider in the past twelve months were associated with HIV testing in the past six months among MSMW in multivariate analyses. One-on-one counseling interventions may increase rates of HIV testing among MSMW.
\end{abstract}

Keywords: HIV counseling, HIV testing, men who have sex with men and women.

\section{BACKGROUND}

In 2006, the Centers for Disease Control (CDC) recommended that sexually active men who have sex with men (MSM) be tested for HIV at least annually [1]. However, recent CDC analyses suggest that MSM should test more frequently. CDC surveillance found that among MSM who had been tested in the past year and who did not report a previous HIV positive test result, 7\% were newly diagnosed seropositive. This high prevalence of new HIV infections among MSM who had been tested during the past year suggests that sexually active MSM might benefit from testing every three to six months [2].

HIV testing is a key component of HIV prevention efforts because knowledge of HIV positivity leads to decreases in HIV transmission [3-4]. HIV testing is also associated with early diagnoses of HIV and, when coupled with appropriate interventions, early diagnosis of HIV has been found to improve health outcomes, including slower clinical progression and reduced mortality [5].

Research suggests that compared to men who have sex with men only (MSMO), men who have sex with men and women (MSMW) are less likely to be tested for HIV [6-8], less likely to be exposed to HIV prevention [8] and more likely to be undiagnosed seropositive $[9,10]$. Despite these findings, very little is known about the HIV testing behaviors of MSMW. Most research on HIV testing among MSM has combined samples of MSMO and MSMW [7, 11-13] or has

*Address correspondence to this author at the Johns Hopkins Bloomberg School of Public Health, Department of Health, Behavior and Society, 624 N. Broadway, Baltimore, MD 21205, USA; Tel: 410-614-3454;

E-mail: cmaulsby@jhsph.edu assessed differences by sexual identity rather than by sexual behavior [14-18]. These studies indicate that HIV testing is associated with factors such as perception of risk, fear of testing positive, structural barriers [13], health status [18], availability of rapid testing, intensive peer counseling [19], socioeconomic factors, race/ethnicity and urban residence [7].

We found no studies that assessed multivariate associations with HIV testing among MSMW. Understanding the factors associated with HIV testing among MSMW provides important information on who is being reached by current HIV testing interventions and how to strengthen current HIV testing initiatives. To address these gaps, this paper 1) describes HIV testing behaviors and use of HIV-related services among MSMW 2) compares HIV testing behaviors and use of HIV-related services of MSMW to MSMO and 3) assesses bivariate and multivariate associations between HIV risk behaviors, use of health services, and HIV testing in the past six months among MSMW.

\section{METHODS}

The National HIV Behavioral Surveillance System (NHBS) is an on-going surveillance system implemented by the CDC that allows for ongoing collection, analysis, and interpretation of HIV risk and prevention behaviors. The Baltimore portion of NHBS is referred to as BESURE and is implemented annually by the Johns Hopkins Bloomberg School of Public Health in collaboration with Maryland Department of Health and Mental Hygiene. BESURE targets three populations at increased risk for HIV: MSM, injection drug users, and heterosexuals. From July to October 2008, the second wave of BESURE-MSM, called BESURE-MSM2, was conducted using venue-based time-space sampling. 
The first step in venue-based sampling is developing a sampling frame. This was done by conducting a series of focus groups with MSM and key stakeholders to identify recruitment venues and corresponding time periods. Venues included bars, restaurants, street locations, festivals, and parks. These venues and times were then randomly selected from the sampling frame to create a sampling events calendar. At these venue and time-specific sampling events, the attendance of all potentially eligible participants was enumerated by counting all individuals who crossed a designated sampling line or area, such as the entrance to a bar. Study recruiters intercepted men who crossed into the sampling area to assess eligibility. Eligibility criteria for men participating in BESURE-MSM2 included (1) being 18 years of age or older, (2) living in Baltimore MSA (metropolitan statistical area), and (3) speaking English or Spanish. MSM behavior was not an eligibility requirement for BESUREMSM2 so as to not bias the study against men who were not comfortable disclosing same sex behaviors at enrollment [20].

Study staff read through the consent form with each participant and addressed any questions. Because the study was anonymous, informed consent was given verbally by the participant and then documented on the consent form by the signature of the study staff member who participated in the consent process. Participants gave informed consent to an interviewer-administered survey and optional HIV test. After consent, eligible participants were given a forty-five minute behavioral risk surveillance survey by trained interviewers and an optional HIV test that included pre-and post-test counseling. Both the survey and the HIV testing took place in a private and secure mobile van. Blood specimens were tested for HIV-1 antibodies with ELISA and a confirmatory Western Blot. After the blood draw, participants scheduled appointments to get their test results and were also given information about BESURE drop-in hours [20].

From July to October 2008, BESURE-MSM2 approached 1,326 men to participate in the study. Sixty-five percent (860) of these men agreed to be screened for eligibility, of these $76 \%$ (654) were eligible, and, of these, $99 \%$ (648) were enrolled. Ninety-three percent of the enrolled participants completed survey and serologic information. Four hundred and forty-eight of enrolled participants reported sex with a man in the past twelve months [20]. Of these 448 men, 43 were known seropositive and were excluded from the analysis, resulting in a final sample of 405 MSM.

\section{Measures}

Men were classified as MSMW if they reported oral, vaginal, or anal sex with a woman in the past twelve months in addition to oral or anal sex with a man in the past twelve months. Men who only reported oral or anal sex with men in the past twelve months were coded as MSMO.

Our outcome of interest, HIV testing within the past six months, was calculated by subtracting the date of the respondent's most recent HIV test from the date of the interview. Date of a respondent's most recent HIV test was obtained by asking "When did you have your most recent HIV test?" Individuals who had never been tested for HIV $(n=43)$ were coded as not having been tested for HIV in the past six months.
Our independent variables of interest included HIV risk behaviors and use of HIV-related services. Number of male sex partners was assessed by asking participants the number of men they had oral or anal sex with in the past year. Unprotected anal sex was assessed by asking "In the past 12 months, with how many of these men did you have anal sex without using a condom?" This question was asked of main, casual, and exchange partners. Having ever injected drugs was assessed by asking, "Have you ever in your life shot up or injected any drugs other than those prescribed for you? By shooting up, I mean any time you might have used drugs with a needle, either by mainlining, skin popping, or muscling" [20].

Participants were asked, "Do you currently have health insurance or coverage? This includes Medicaid or Medicare" and then asked, "What kind of health insurance or coverage do you currently have?" Access to a health care provider was assessed by asking, "Have you seen a doctor, nurse, or other health care provider in the past 12 months?"

Disclosure of same sex behavior to a health care provider was assessed by asking two questions. Participants were first asked, "Have you ever told anyone that you are attracted to or have sex with men?" Individuals who gave a positive response were then asked "I'm going to read you a list of people you may have told. Please tell me which ones apply. Have you told: a health care provider."

To assess if participants had been exposed to one-to-one HIV counseling services, participants were asked: "In the past 12 months, have you had a one-on-one conversation with an outreach worker, counselor, or prevention program worker about ways to prevent HIV? Don't count the times when you had a conversation as part of an HIV test." Conversations that were part of an HIV test were excluded to not artificially inflate a positive relationship between counseling and HIV testing.

\section{Statistical Analysis}

We investigated the relationship between frequency of venue attendance and our outcome of interest, HIV testing in the past six months, to assess the need for weighted estimates. Men with higher attendance might be more likely to be enrolled in the study. Therefore, if venue attendance was associated with our outcome, our results would be biased. We found that venue attendance was not associated with HIV testing, and therefore we did not weight our data to account for frequency of venue attendance [21].

Differences between MSMW and MSMO in use of HIVrelated services were evaluated using Pearson's Chi square for categorical variables. Fisher's exact test was executed when cell sizes fell below five.

Unadjusted prevalence ratios (PR), adjusted prevalence ratios (APR) and corresponding 95\% confidence intervals (95\% CI) were calculated using SAS PROC GENMOD's log-binomial regression capability with a binomial distribution and a logarithmic link function [22]. For unadjusted and adjusted analyses, we used the REPEATED statement to account for clustering by venue. For multivariate analyses, we employed the COPY method if the log-binomial model did not converge [23]. Our multivariate analysis only included variables with a univariate association 
with HIV testing of $p \leq 0.10$. We used prevalence ratios rather than odds ratios to approximate relative risk because the frequency of our outcome of interest, HIV testing, exceeded $10 \%$ [24]. We arrived at the most parsimonious model by removing variables that were insignificant $(p>$ 0.05 ) using a stepwise approach. Model fit was determined using QIC (Quasi-likelihood under the Independence model Criterion). QIC is a method used to compare two GEE models to determine the model with the best fit. The model with the smaller QIC statistic is preferred. All statistical analyses were performed using Statistical Analysis Software (SAS) version 9.1 (SAS Institute, Cary, NC).

\section{RESULTS}

\section{Participant Characteristics and Use of HIV-Related Services}

The demographic characteristics of our study population has been reported elsewhere [20]. In brief, 300 (74.1\%) MSM reported sex with men only and $105(25.9 \%)$ reported sex with both men and women. The majority of the sample was African
American (70.9\%), under the age of $35(63.0 \%)$, had some college education (52.1\%), and identified as homosexual (66.8\%) (Table 1). As reported previously, compared to MSMO, MSMW were more likely to be African American, be homeless, have less than a high school education, be in poverty, and identify as bisexual, heterosexual or other [20].

Table 2 outlines differences between MSMW and MSMO in use of HIV-related health services. MSMW were more likely than MSMO to report no insurance (48.1\% versus $34.4 \%, p=.0132$ ) and to report public insurance (28.9\% versus $14.6 \%, p=.0130)$. We found that MSMW were significantly less likely than MSMO to have ever disclosed their same sex behavior to a health care provider $(49.5 \%$ versus $71.8 \%, p=<.0001)$ and to have heard of local organizations that provide HIV programs $(33.0 \%$ versus $54.9 \%, p=0.0001)$. MSMW were as likely as MSMO to have visited a health care provider in the past year and to attend a one-on-one HIV counseling session.

About half of the MSM in our study had been tested for HIV in the past year (MSMO 56.0\%, MSMW 47.6\%,

Table 1. Socio-Demographic Characteristics of MSMO and MSMW (n=405)

\begin{tabular}{|c|c|c|c|c|}
\hline & Total & $\frac{\text { MSM/O }}{\text { (Column \%) }}$ & $\frac{\text { MSM/W }}{\text { (Column \%) }}$ & P-Value \\
\hline Total & 405 & $300(74.1)$ & $105(25.9)$ & \\
\hline \multicolumn{5}{|l|}{ Race } \\
\hline White & $94(23.2)$ & $85(28.3)$ & $9(8.6)$ & $<.0001$ \\
\hline Other & $24(5.9)$ & $18(6.0)$ & $6(5.7)$ & 0.9150 \\
\hline \multicolumn{5}{|l|}{ Age } \\
\hline$<24$ years old & $134(33.1)$ & $107(35.7)$ & $27(25.7)$ & 0.0621 \\
\hline \multicolumn{5}{|l|}{ Current Homeless } \\
\hline No & $381(94.1)$ & $292(97.3)$ & $89(84.8)$ & $<0.0001$ \\
\hline Yes & $24(5.9)$ & $8(2.7)$ & $16(15.2)$ & $<0.0001$ \\
\hline \multicolumn{5}{|l|}{ Education } \\
\hline High school or less & $194(47.9)$ & $128(42.7)$ & $66(62.9)$ & 0.0004 \\
\hline College or some college & $187(46.2)$ & $151(50.3)$ & $36(34.3)$ & 0.0045 \\
\hline Graduate education & $24(5.9)$ & $21(7.0)$ & $3(2.9)$ & 0.1218 \\
\hline \multicolumn{5}{|l|}{ Poverty } \\
\hline Heterosexual/other & $9(2.2)$ & $2(0.7)$ & $7(6.7)$ & 0.0003 \\
\hline
\end{tabular}


Table 2. Differences in Use of Health Services Related to HIV Testing Between MSMO and MSMW (N=405)

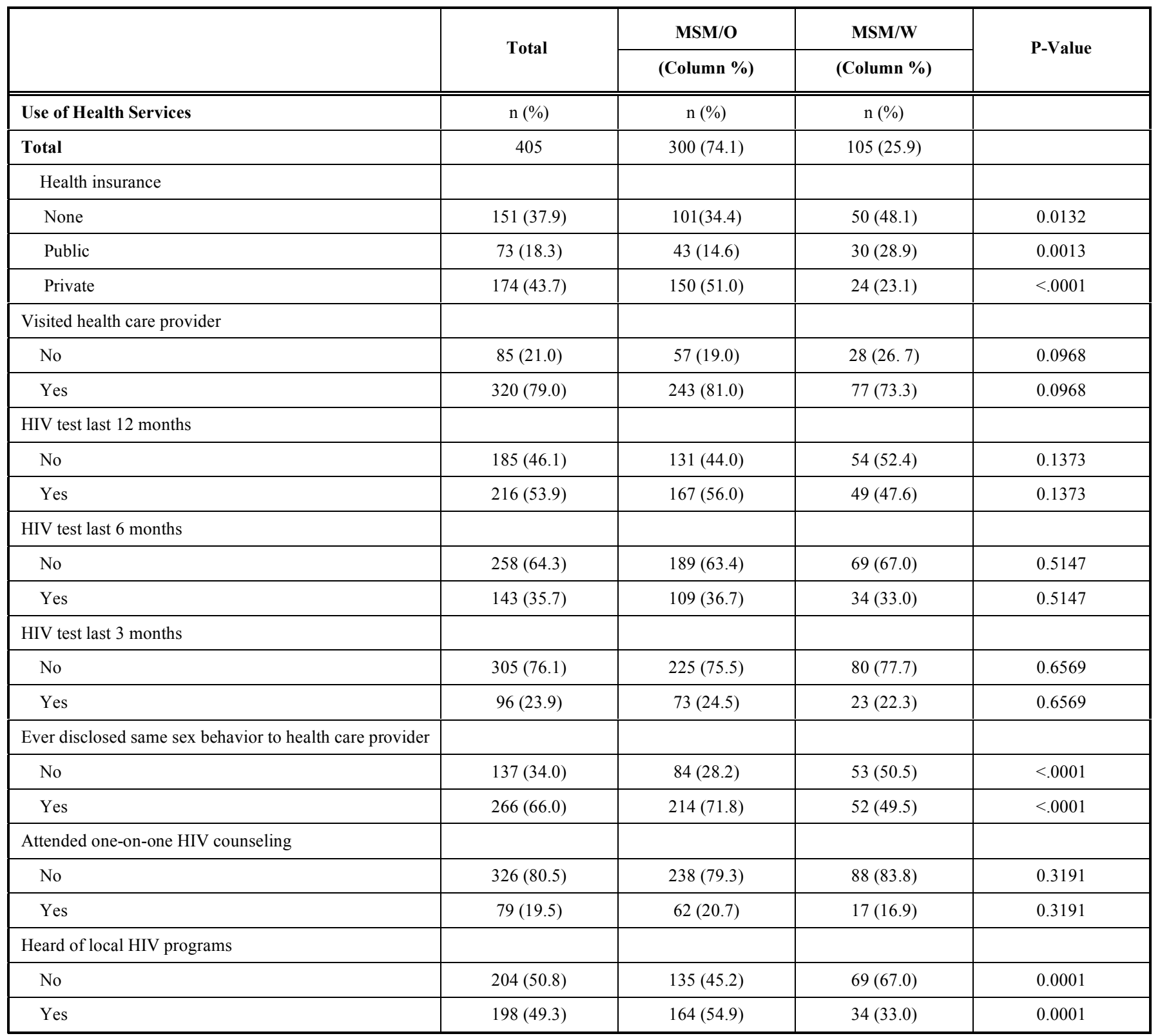

$p=.1373$ ), and about a third had been tested in the past six months (MSMO 36.7\%, MSMW 33.0\%, $p=.5147$ ). MSMO and MSMW were equally likely to have been tested for HIV in the past three months. When we looked at differences in risk behavior among those who had and had not been tested, we found no differences in unprotected anal intercourse (UAI) with men in the past year, UAI with men at last sex, or in number of male sex partners. We also found no differences when we stratified this analysis by MSMO and MSMW (results not shown).

Table 3 highlights associations between HIV risk behaviors, use of health services and HIV testing among MSMW. In bivariate analyses race/ethnicity, having unprotected sex with a female partner in the past year, having been to a health care provider in the past year, and attending one-on-one counseling were associated with HIV testing at the $p<.10$ level. In multivariate analyses MSMW who had been to a health care provider in the past year were three times more likely to have been tested for HIV in the past six months (APR $=3.1 ; 95 \% \mathrm{CI}=1.3-7.6)$, and MSMW who had attended an one-on-one counseling session were two times more likely to have been tested for HIV in the past six months $(\mathrm{APR}=2.2 ; 95 \% \mathrm{CI}=1.2-4.1)$. The $\mathrm{QIC}$ for the final model was 112.8. To assess if HIV predictive factors differed by MSMO/MSMW status, we looked at predictors of HIV testing among the total population of 405 MSM with MSMO or MSMW status included as an interaction term. We found that for the combined sample, MSM who had visited a health care provider in the past year were 1.3 times as likely to have been tested for HIV in the past six months $(\mathrm{APR}=1.3 ; 95 \% \mathrm{CI}=1.0-1.7)$, and that MSM who had attended a one-on-one counseling session were 2.0 times as likely to have been tested for HIV in the past six months $(\mathrm{APR}=2.0 ; 95 \% \mathrm{CI}=1.2-3.2)$ (results not displayed). When we tested for interactions by MSMW status we found that neither the relationship between visiting a health care 
Table 3. Associations with HIV Testing in the Past Six Months Among MSMW (N=105)

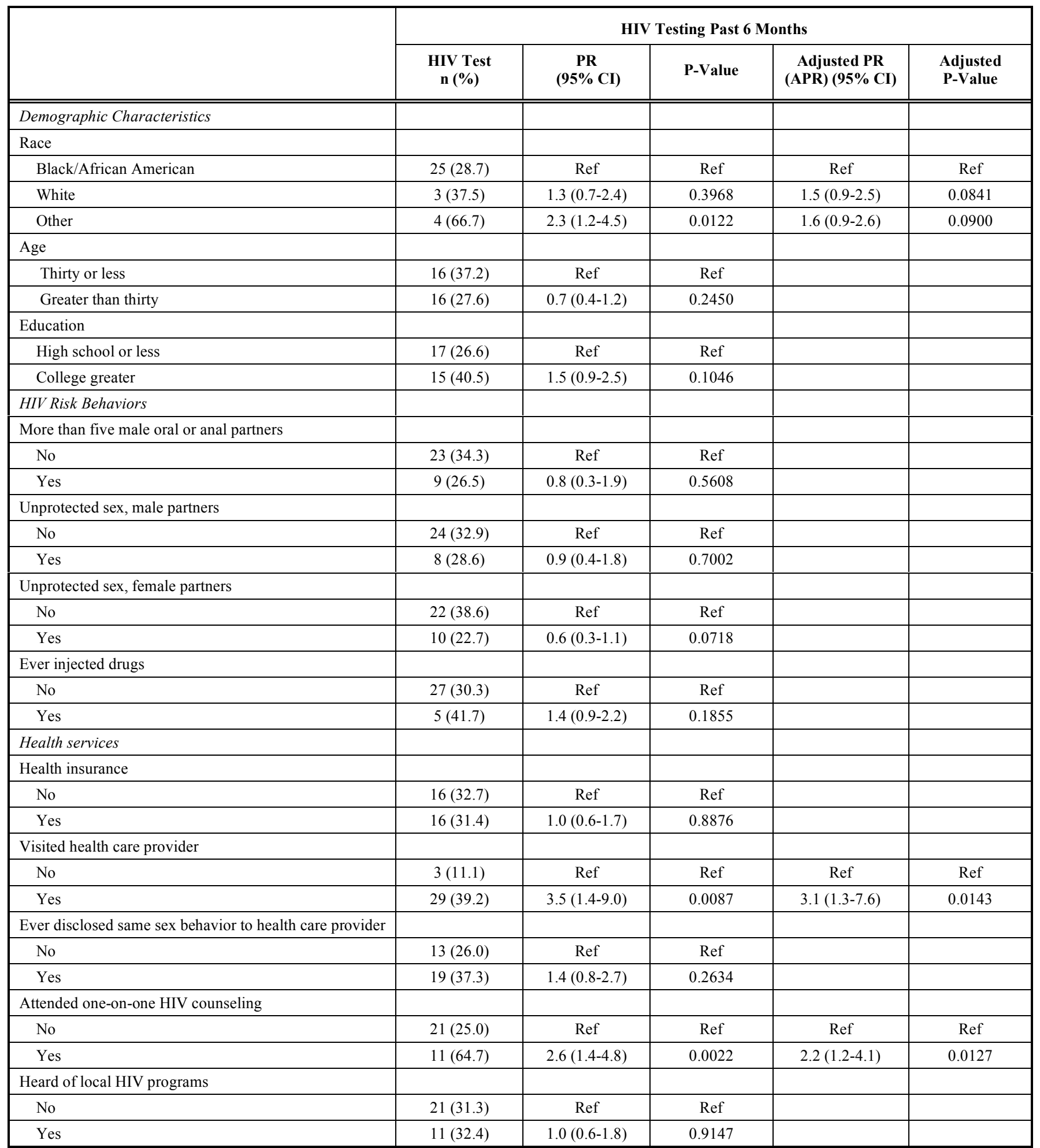

provide and HIV testing $(p=0.3467)$ nor the relationship between one-on-one counseling and HIV testing ( $p=0.0701)$ varied across MSMO/MSMW strata (results not displayed).

\section{DISCUSSION}

When we looked at differences between MSMO and MSMW in likelihood of testing, we found that MSMO and
MSMW were equally likely to have been tested in the past three, six, and twelve months. Previous research has found that MSMW were less likely than MSMO to have been tested ever [6, 8, 25] or in the past five years [26]. Our finding that MSMO and MSMW are equally likely to have been tested is encouraging. The implementation of HIV screening in health care settings combined with testing in non-clinical settings such as mobile vans and shelters might 
help to explain these findings. Because we do not have information on where respondents were tested, we were not able to assess if MSMW and MSMO are being testing in the same locations. This represents an area in need of further study.

Our finding that MSMW were less likely to disclose same-sex behavior to a health care provider (HCP) than MSMO supports earlier research [27, 28]. In bivariate analyses our data indicated a positive but non-significant relationship between disclosing same-sex behavior to a HCP and HIV testing among MSMW. Past research has found that disclosure of same-sex behavior was positively associated with HIV testing [6, 27, 28]. Theory suggests that decisionmaking about disclosure of same-sex behavior is driven by the characteristics of the health care provider, the health care environment, and the individual considering disclosure. Factors that inhibit disclosure of same-sex behavior include: fear of poor care, discrimination, rejection, hostility and judgment from the provider, perceptions that the provider may not be able "to handle" the subject matter or that the provider might be uncomfortable with same-sex behavior, and concerns about confidentiality [28-30].

In multivariate analyses, we found that attending a oneon-one HIV counseling session was associated with HIV testing. Previous research suggests that counseling is an effective intervention for health behavior change among MSM [31-34]. Our findings suggest that one-on-one counseling that is not part of HIV testing is positively associated with HIV testing, and therefore might be a useful strategy for increasing HIV testing in Baltimore. In Baltimore, the prevalence of undiagnosed seropositivity among HIV positive MSMW is estimated to be $87.9 \%$ [10]. We found that predictors of HIV testing did not differ for MSMO and MSMW populations. However, the unique needs of MSMW and the degree of marginalization that this population faces, warrant tailored HIV testing and prevention interventions.

Our study has multiple limitations. First, the behavioral study data was self-reported by participants and the validity of self-report data has been questioned. Participants may have underreported stigmatized behaviors and over reported normative behaviors [35]. A literature review conducted by National Institutes of Health in 1998 found that welldesigned interviews and questionnaires can provide acceptable data when administered appropriately [36]. Steps recommended in this article were followed during the implementation of BESURE-MSM2, such as conducting formative research, using easy to understand language, and using time sequence techniques. An additional limitation of this study is that it is cross-sectional and does not assess causality. The sample size for the analyses presented in this paper is small, which might have limited our ability to detect significant differences. We would like to note that the confidence intervals for visiting a health care provider and attending a one-to-one HIV counseling session in our multivariate analysis are wide, which might suggest that our sample population was not large enough. Finally, individuals who opted to not participate in BESURE might be systematically different from those who did. For example, participants who agreed to participant in the study might be of lower income that individuals who did not participate in the study, resulting in selection bias.

The findings from this study represent MSMW and MSMO over 18 who live in Baltimore, who attend the type venues included in this study (bars, parks, and street corners) and who are not known HIV seropositive. Generalizability beyond these characteristics and settings is unknown [20].

Despite the study limitations, our findings have important implications for future research and for HIV prevention programs. Further research is needed to better understand the factors, including social contextual factors that influence HIV testing, knowing one's HIV results and delayed HIV diagnosis among MSMW. One-on-one HIV counseling prevention programs might be an effective strategy to increase HIV testing among MSMW, a highly marginalized sub-population of MSM at increased risk for undiagnosed seropositivity.

\section{ACKNOWLEDGEMENTS}

The authors express their gratitude to the BESURE Study field staff for their dedication and to the men who participated in this study. The BESURE study was supported by contracts to The Johns Hopkins University from the Maryland Department of Health and Mental Hygiene and by cooperative agreements between the Maryland Department of Health and Mental Hygiene with the Centers for Disease Control and Prevention. The analyses described here were supported by Award Number F31MH084767 from the National Institutes of Mental Health. The authors also acknowledge the Mentoring Program of The Center for Population Research in LGBT Health, supported by the Eunice Kennedy Shriver National Institute of Child Health and Human Development (NICHD) under Award Number R21HD051178. The content is solely the responsibility of the author(s) and does not necessarily represent the official views of the NICHD, the National Institutes of Mental Health or the National Institutes of Health. We thank Laura Wehrmeyer for her assistance with copy editing this publication.

\section{CONFLICTS OF INTEREST}

The authors have no conflicts of interest to report.

\section{REFERENCES}

[1] Branson BM, Handsfield HH, Lampe MA, et al. Revised recommendations for HIV testing of adults, adolescents, and pregnant women in health-care settings. MMWR Recomm Rep 2006; 55: 1-17.

[2] CDC. HIV Testing among men who have sex with men --- 21 cities, United States, 2008. Morb Mortal Wkly Rep (MMWR) 2011; 60: 694-9.

[3] Marks G, Crepaz N, Janssen RS. Estimating sexual transmission of HIV from persons aware and unaware that they are infected with the virus in the USA. AIDS 2006; 20: 1447-50.

[4] Holtgrave DR, Pinkerton SD. Can increasing awareness of HIV seropositivity reduce infections by $50 \%$ in the United States? J Acquir Immune Defic Syndr 2007; 44: 360-3.

[5] US Preventive Services Task Force. Screening for HIV: recommendation statement. Ann Intern Med 2005; 143: 32-7.

[6] Daskalakis D, Silvera R, Bernstein K, et al. Implementation of HIV testing at 2 New York city bathhouses: from pilot to clincal service. Clin Infect Dis 2009; 48: 1609-16.

[7] Jeffries WL. HIV testing among bisexual men in the United States. AIDS Educ Prev 2010; 22: 356-70. 
[8] Flores SA, Bakeman R, Millett GA, Peterson JL. HIV risk among bisexually and homosexually active racially diverse young men. Sex Transm Dis 2009; 36: 325-9.

[9] Young SD, Shoptaw S, Weiss RE, Munjas B, Gorbach PM. Predictors of unrecognized hiv infection among poor and ethnic men who have sex with men in Los Angeles. AIDS Behav 2009; 15: 643-9.

[10] German D, Sifakis F, Maulsby C, et al. Persistently high prevalence and unrecognized HIV infection among men who have sex with men in Baltimore: the BESURE Study. J Acquir Immune Defic Syndr 2011; 57: 77-87.

[11] Sanchez T, Finlayson T, Drake A, et al. Human immunodeficiency virus (HIV) risk, prevention, and testing behaviors--United States, National HIV Behavioral Surveillance System: men who have sex with men, November 2003-April 2005. MMWR Surveill Summ 2006; 55: 1-16.

[12] Helms DJ, Weinstock HS, Mahle KC, et al. HIV testing frequency among men who have sex with men attending sexually transmitted disease clinics: implications for HIV prevention and surveillance. J Acquir Immune Defic Syndr 2009; 50: 320-6.

[13] Mackellar DA, Hou SI, Whalen CC, et al. Reasons for not HIV testing, testing intentions, and potential use of an over-the-counter rapid HIV test in an internet sample of men who have sex with men who have never tested for HIV. Sex Transm Dis 2011; 38: 419-28.

[14] Jin FY, Prestage G, Law MG, et al. Predictors of recent HIV testing in homosexual men in Australia. HIV Med 2002; 3: 271-6.

[15] Lauby JL, Milnamow M. Where MSM have their first HIV test: differences by race, income, and sexual identity. Am J Mens Health 2009; 3: 50-9.

[16] Pathela P, Hajat A, Schillinger J, et al. Discordance between sexual behavior and self-reported sexual identity: a population-based survey of New York City men. Ann Intern Med 2006; 145: 416-25.

[17] Wolitski RJ, Jones KT, Wasserman JL, Smith JC. Selfidentification as "down low" among men who have sex with men (MSM) from 12 US cities. AIDS Behav 2006; 10: 519-29.

[18] Mimiaga MJ, Landers SJ, Conron KJ. Prevalence and correlates of lifetime HIV testing in a population-based sample of men who have sex with men in massachusetts. AIDS Patient Care STDS 2011; 25: 323-6.

[19] Lorenc T, Marrero GI, Aggleton P, et al. Promoting the uptake of HIV testing among men who have sex with men: systematic review of effectiveness and cost-effectiveness. Sex Transm Infect 2011; 87: 272-8.

[20] Maulsby C, Sifakis F, German D, Flynn CP, Holtgrave D. Partner characteristics and undiagnosed HIV seropositivity among men who have sex with men only (MSMO) and men who have sex with men and women (MSMW) in Baltimore. AIDS Behav 2011; 16: 543-53.

[21] MacKellar D, Valleroy L, Karon J, Lemp G, Janssen R. The Young Men's Survey: methods for estimating HIV seroprevalence and risk factors among young men who have sex with men. Public Health Rep 1996; 111 (Suppl 1): 138-44.
[22] Spiegelman D, Hertzmark E. Easy SAS calculations for risk or prevalence ratios and differences. Am J Epidemiol 2005; 162: 199200 .

[23] Deddens JA, Petersen MR. Approaches for estimating prevalence ratios. Occup Environ Med 2008; 65: 501-6.

[24] McNutt LA, Wu C, Xue X, Hafner JP. Estimating the relative risk in cohort studies and clinical trials of common outcomes. Am J Epidemiol 2003; 157: 940-3.

[25] Wheeler DP, Lauby JL, Liu KL, Van Sluytman LG, Murrill C. A comparative analysis of sexual risk characteristics of Black men who have sex with men or with men and women. Arch Sex Behav 2008; 37: 697-707.

[26] Mercer CH, Hart GJ, Johnson AM, Cassell JA. Behaviourally bisexual men as a bridge population for HIV and sexually transmitted infections? Evidence from a national probability survey. Int J STD AIDS 2009; 20: 87-94.

[27] Bernstein KT, Liu KL, Begier EM, et al. Same-sex attraction disclosure to health care providers among New York City men who have sex with men: implications for HIV testing approaches. Arch Intern Med 2008; 168: 1458-64.

[28] Johnson CV, Mimiaga MJ, Reisner SL, et al. Health care access and sexually transmitted infection screening frequency among atrisk Massachusetts men who have sex with men. Am J Public Health 2009; 99 (Suppl 1): S187-92.

[29] Eliason M, Schope R. Does "Don't ask don't tell" apply to health care? lesbian, gay, and bisexual people's disclosure to health care providers. J Gay Lesbian Med Assoc 2001; 5: 125-43.

[30] Mimiaga MJ, Goldhammer H, Belanoff C, Tetu AM, Mayer KH. Men who have sex with men: perceptions about sexual risk, HIV and sexually transmitted disease testing, and provider communication. Sex Transm Dis 2007; 34(2): 113-9.

[31] Dilley JW, Woods WJ, Sabatino J, et al. Changing sexual behavior among gay male repeat testers for HIV: a randomized, controlled trial of a single-session intervention. J Acquir Immune Defic Syndr 2002; 30: 177-86.

[32] Kamb ML, Fishbein M, Douglas JM Jr, et al. Efficacy of riskreduction counseling to prevent human immunodeficiency virus and sexually transmitted diseases: a randomized controlled trial. Project RESPECT Study Group. JAMA 1998; 280: 1161-7.

[33] Koblin B, Chesney M, Coates T. Effects of behavioural interventions to reduce acquisition of HIV infection among men who have sex with men: the EXPLORE randomised controlled study. The Lancet 2004; 364: 41-50.

[34] Picciano JF, Rothman RA, Kalichman SC, Rutledge SE, Berghuis JP. A telephone based brief intervention using motivational enhancement to facilitate HIV risk reduction among MSM: A pilot study. AIDS Behav 2001; 5: 251-62.

[35] Lewontin RC. Sex, lies and social science. New York Review of Books 1995; pp. 24-9.

[36] Weinhardt LS, Forsyth AD, Carey MP, Jaworski BC, Durant LE. Reliability and validity of self-report measures of HIV-related sexual behavior: progress since 1990 and recommendations for research and practice. Arch Sex Behav 1998; 27: 155-80. 